\title{
Pantarsal Arthrodesis to Treat a Full-Thickness Lateral Trochlear Ridge Osteochondritis Dissecans in a Dog
}

\author{
Petazzoni Massimo ${ }^{1}$ Buiatti Michela ${ }^{1}$ \\ ${ }^{1}$ Department of Orthopaedics and Traumatology, Clinica Veterinaria \\ Milano Sud (MI), Peschiera Borromeo, MI, Italy \\ Address for correspondence Petazzoni Massimo, DVM, Department \\ of Orthopaedics and Traumatology, Clinica Veterinaria Milano Sud \\ (MI), Via Liberazione 26, Peschiera Borromeo, MI 20068, Italy \\ VCOT Open 2019;2:e27-e31. \\ (e-mail: massimo.petazzoni@cvmilanosud.it).
}

\begin{abstract}
Keywords

- osteochondritis dissecans

- hock

- arthrodesis

- locking plate

A 5-month-old female Golden Retriever dog presented with a 4-week history of right hindlimb lameness. A bone lesion on the proximal lateral trochlear ridge was detected at radiographic and ultrasound examinations. Osteochondritis dissecans (OCD) or an articular fracture was included in the differential diagnoses. Computed tomography revealed an OCD lesion. A pantarsal arthrodesis was performed using a pre-bent arthrodesis locking plate. Histological analysis revealed pathological features typical of OCD. Clinical and radiographical assessments were performed 7, 15, 62 days and 7, 12 months postoperatively. No signs of pain were elicited upon palpation. Mechanical lameness, secondary to tarsal joint immobilization, was detected.
\end{abstract}

\section{Introduction}

Osteochondritis is a growth disorder in which there is altered endochondral ossification which causes thickening and retention of a circumscribed area of cartilage. ${ }^{1,2}$ Osteochondritis evolves into osteochondritis dissecans (OCD) when the part of altered cartilage becomes detached from the subchondral bone, causing a joint flap. ${ }^{3}$ The hock is the third joint most frequently affected by OCD, after the shoulder and elbow ${ }^{2}$ and is the site of 4 to $9 \%$ of all cases of OCD. ${ }^{4}$ Among dogs, it affects animals between 4 and 9 months of age, ${ }^{5}$ with a predilection for females and for the Rottweiler and Labrador Retriever breeds. ${ }^{6,7}$ In $79 \%$ of cases of OCD of the hock the medial border of the talar trochlea is affected, ${ }^{2}$ while in the remaining $21 \%$ of the cases it is the lateral border which is affected (of which 70\% of cases involve the dorsal part of the trochlear ridge). ${ }^{8,9}$ The surgical treatment consists of removing the fragment of detached cartilage via arthroscopy or arthrotomy. ${ }^{10,11}$ In $24 \%$ of the cases treated surgically, by removal of the joint flap, the clinical outcome is judged unsatisfactory, particularly when the flap is large, while in $42 \%$ of cases the patient has persistent, chronic lameness. ${ }^{6,12}$ The use of a synthetic osteochondral resurfacing implant for the treatment of OCD of the femoral condyle was reported as a successful and repeatable surgical procedure in selected patients. ${ }^{13}$ Fractures of the talar trochlea are rare and poorly described in the literature. ${ }^{14-16}$ The recommended treatment for simple fractures of the lateral ridge of the talar trochlea is anatomical reduction in the joint fragment and fixation with screws or Kirschner wires ${ }^{15,16}$; if the fracture cannot be reduced, arthrodesis is recommended. ${ }^{17}$ Pantarsal arthrodesis of the tarsus is considered in the literature as a limb-saving intervention in cases of chronic osteoarthritis, rupture of the plantar ligament, rupture of the calcaneal ligament, irreducible joint fractures, or traumatic tibiotarsal dislocation. ${ }^{17,18}$ A clinical case of full-thickness OCD of the lateral trochlear ridge of the talus managed with pantarsal arthrodesis is described.

\section{Case Report}

A 5-month-old, female Golden Retriever weighing $30 \mathrm{~kg}$ was brought for the clinical evaluation of right hindlimb lameness. The reported history was a sudden onset of limping and partial weight-bearing which developed secondary to a traumatic event (running in the park) and had become chronic (4 weeks). The orthopaedic evaluation revealed pain on deep palpation of the tibiotarsal region. The differential diagnoses included a fracture, a sprain or a hock OCD. The dog was given general anaesthesia to take X-rays of the received

November 1, 2018 accepted after revision March 7, 2019
DOI https://doi.org/

10.1055/s-0039-1687885. ISSN 2625-2325. (c) 2019 Georg Thieme Verlag KG
Stuttgart · New York

License terms

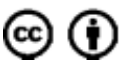




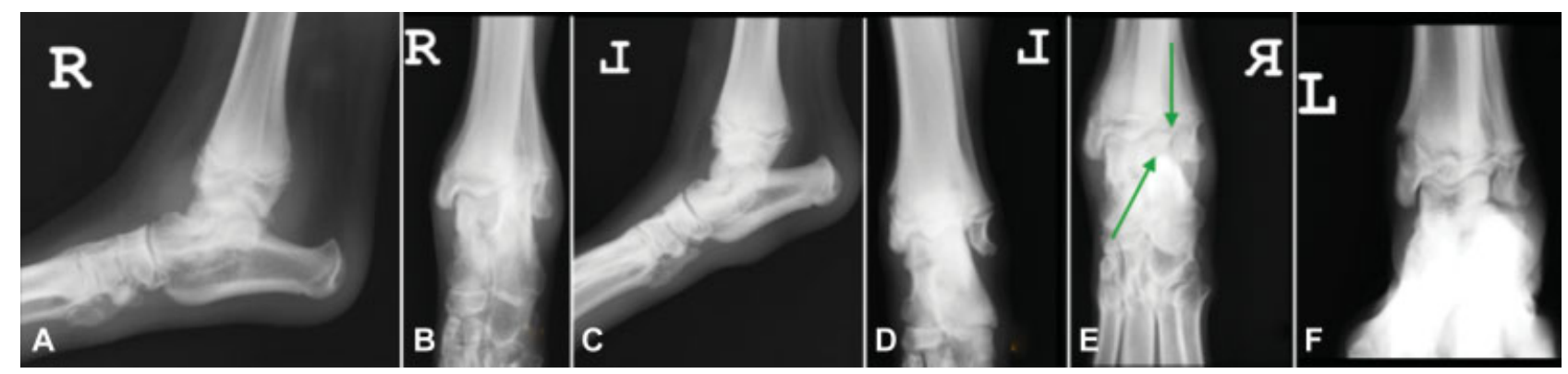

Fig. 1 Preoperative X-rays. (A) Mediolateral and (B) anteroposterior views of the right, affected limb. (C) Mediolateral and (D) posteroanterior projections of the left, unaffected limb. Craniocaudal axial X-rays of the (E) right hindlimb and the (F) left hindlimb. The X-ray shows a lesion in the dorsolateral aspect of the lateral ridge of the talus (green arrow).

hindlimbs. The radiographic evaluation included mediolateral and dorsoplantar projections and a craniocaudal axial view of the talar trochlea with the tibiotalar joint flexed at $90^{\circ}$ (-Fig. 1). The craniocaudal axial X-ray showed tibiotarsal joint space narrowing, discontinuity of the subchondral bone of the lateral trochlear ridge of the talus and a fragment of fractured bone corresponding to the dorsal aspect of the lateral talar ridge (-Fig. 1). A subsequent ultrasound examination detected joint effusion and a fracture in the subchondral bone of the lateral ridge of the tarsal trochlea (-Fig. 2). A computed tomography (CT) study of both distal hindlimbs was performed to understand the lesion better and determine its extension. The CT images revealed an articular flap with the characteristic appearance of OCD (-Fig. 3). Ten days later, a pantarsal arthrodesis was performed (-Fig. 4A).

The patient was placed in dorsal recumbency and a dorsal surgical access to the tibiotarsal joint was performed. The osteochondral fragment was removed and sent to a veterinary laboratory for the histological examination (-Fig. 5). Cartilage and subchondral bone of the remaining part of the lateral tarsal ridge, on the tibial side, were removed by a high-speed bur $(100,000 \mathrm{~K})$ and an oscillating saw (Command 2 blade 5400-3-410; Stryker, Formello, RM, Italy). Subsequently, the

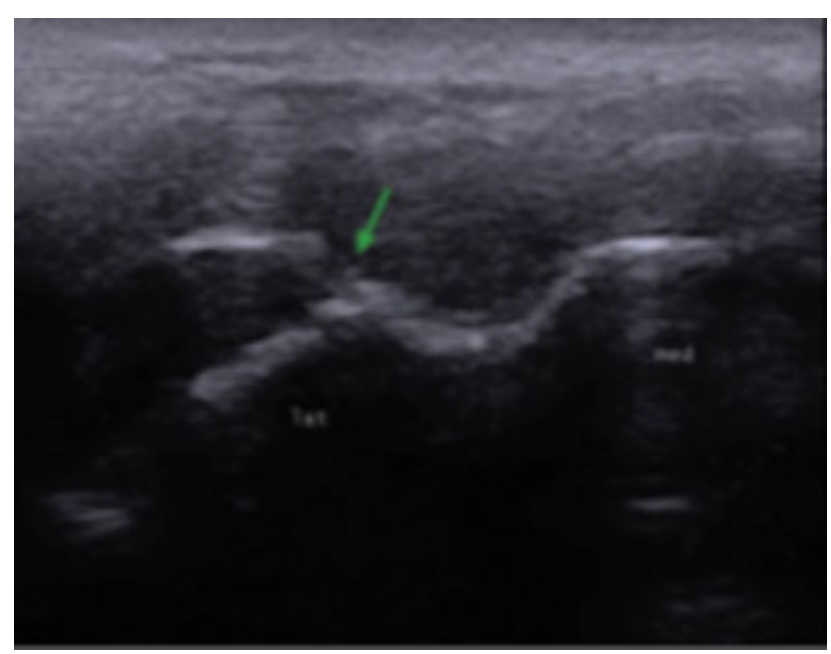

Fig. 2 Ultrasound image. Transverse section of the tarsus in extension at the level of the lateral and medial borders of the talar trochlea. Note the break in the lateral ridge of the talar trochlea (arrow). distal cartilage of the tarsus, calcaneus, proximal side of the central bone of the tarsus and the fourth tarsal bone down to the underlying subchondral bone were removed by a highspeed bur. The intratarsal and tarsometatarsal cartilage was removed by burring as well. The spaces created by the removal of the fragment, the cartilage and subchondral bone were filled with $2 \mathrm{~mL}$ of Fortigen P (Progenica Therapeutics; Kent, Washington, United States). For the purpose of stabilizing the joint, an angular, Fixin locking plate (Intrauma Ref. V3011, Rivoli, TO, Italy), pre-bent at $135^{\circ}$ (120 mm long and $2.5 \mathrm{~mm}$ thick) was placed with eight screws: two $3.5-\mathrm{mm}$ locking screws and one $3.5-\mathrm{mm}$ compression screw in the distal tibia, two $3.0 \mathrm{~mm}$ locking screws into the talus and third tarsal bone, two 3.0-mm locking screws in the central and distal thirds of the third metatarsal bone and one $2.7-\mathrm{mm}$ nonlocking screw in the proximal third of the third metatarsal bone. Two X-rays were performed, one in a mediolateral projection and the other in a posteroanterior projection, to check the positions of the implants and the alignment of the $\operatorname{limb}$ (-Fig. 4A).

A light Robert Jones bandage was applied for 5 days, extending from the distal femur to include the phalanges. The dog was discharged with an antibiotic prescription (amoxicillin + clavulanic acid, $20 \mathrm{mg} / \mathrm{kg}$ twice daily [BID]) for 10 days, anti-inflammatory therapy (robenacoxib $1 \mathrm{mg} /$ $\mathrm{kg}$ once daily) for 3 weeks and analgesia (tramadol $2 \mathrm{mg} / \mathrm{kg}$ BID) for 3 weeks. Cage confinement was suggested until radiographic confirmation of bone healing. Leash walking was limited to four times a day, for 10 minutes each. Clinical examinations were performed 7, 15 and 62 days after surgery. At day 7 and 15 evident lameness was detected. At 62 days the joint was stable and not painful on palpation, although walking was abnormal, with external rotation of the pes. At 62 days from surgery the radiographic follow-up revealed bone fusion ( - Fig. 4B). Seven months after surgery, the patient was brought for a re-evaluation because of slight lameness. Moderate pain was elicited upon deep palpation of the distal tibia. A mediolateral X-ray was performed. Radiolucent areas were detected in the distal tibia and at the base of the metatarsal bones (-Fig. $4 \mathbf{4 C}$ ). The implants were removed. The patient was referred for a clinical and radiographic control 1 year after surgery, at which time the joint was stable and not painful on palpation (-Fig. 4D). Walking and trotting were abnormal with external rotation of the pes. 


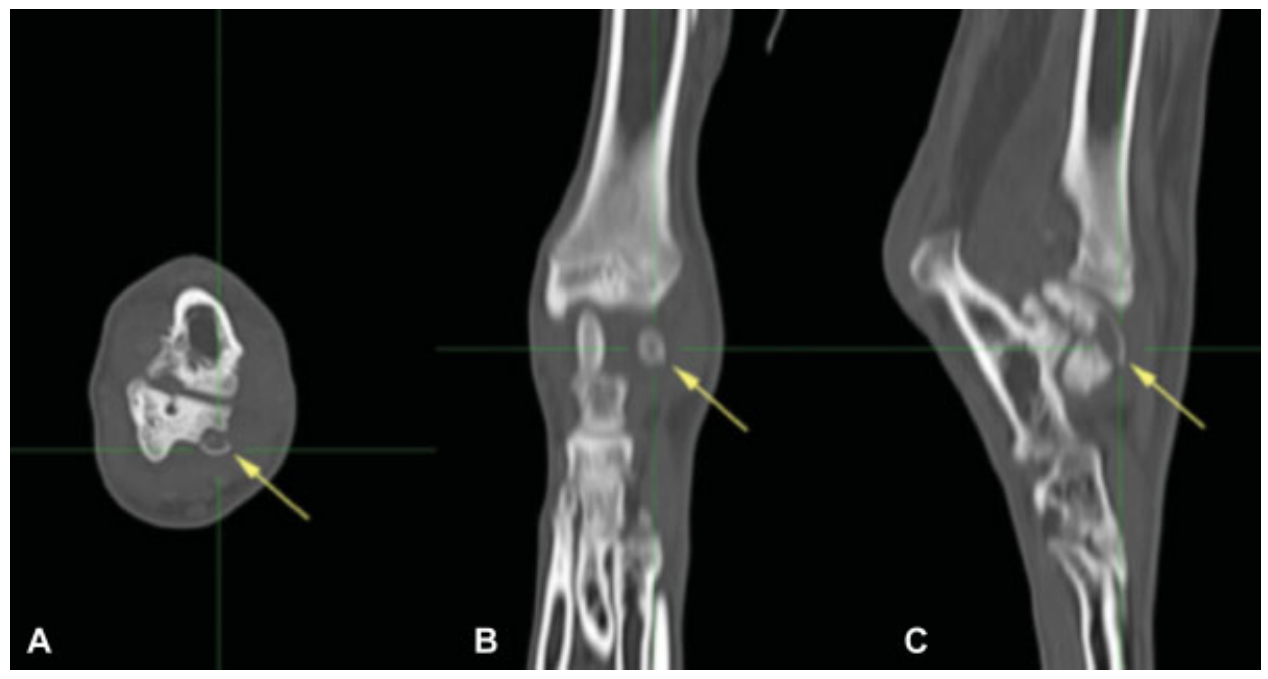

Fig. 3 Computed tomography multiplanar reconstruction images of the right limb. The arrows point to the osteochondritis dissecans lesions of the right talus on the transverse (A), frontal (B) and sagittal (C) planes. Periarticular moderate soft tissue swelling can be detected.

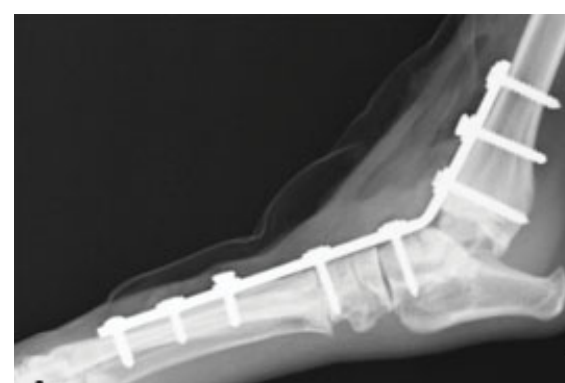

A
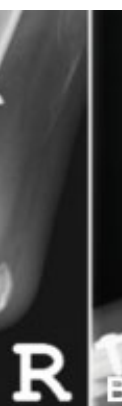
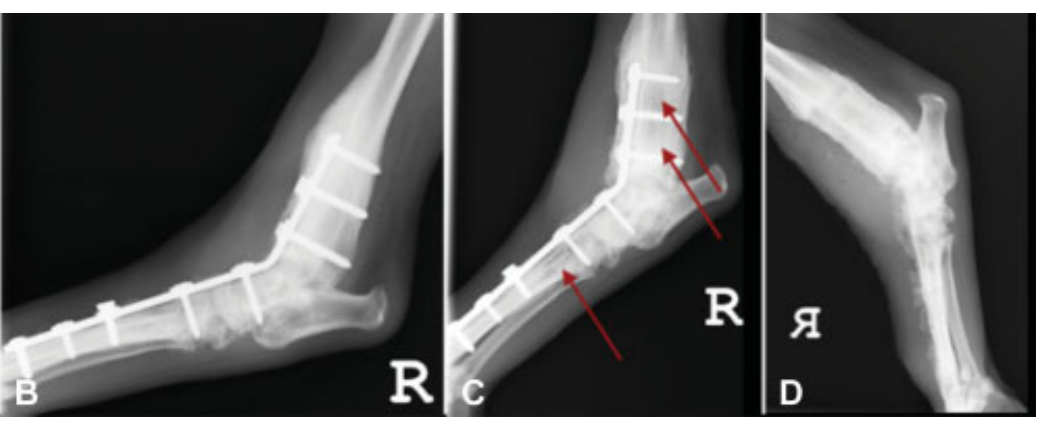

Fig. 4 (A) Postoperative, (B) 62 days, (C) 7 months and (D) 1 year X-rays in mediolateral view. X-ray control 62 days after surgery, showing radiographic healing: note the exuberant callous in the tibial portion of the plate. At 7 months after surgery: the red arrows highlight areas of radiolucency that could indicate a stress protection phenomenon.

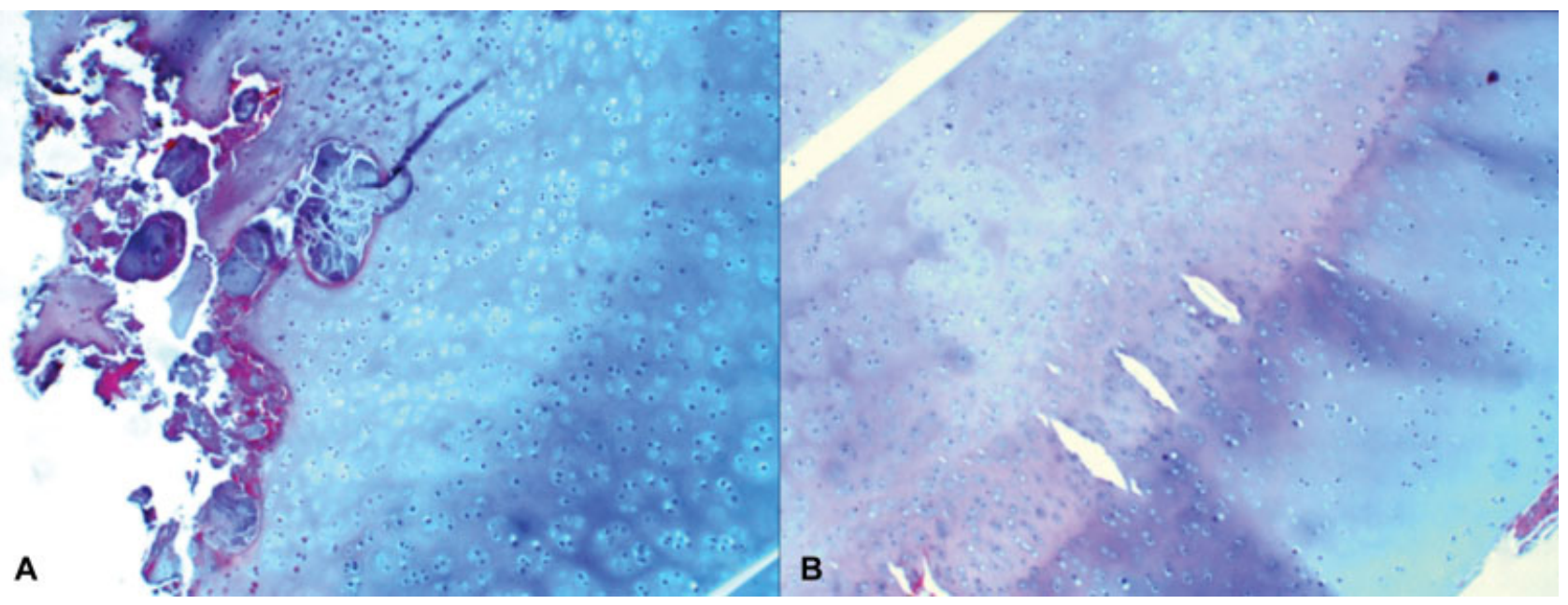

Fig. 5 Histological images of the osteocartilage joint fragment: a large area of vascular necrosis surrounded by immature, hypertrophic chondrocytes, often binucleated and arranged in small, irregular aggregates embedded in scarce deposits of amorphous chondroid matrix is observed. The alterations to the cartilage are compatible with osteochondritis dissecans. Staining technique Alcian blue periodic acid Schiff magnification 10X (A). Staining technique Alcian blue periodic acid Schiff magnification 40X (B).

\section{Discussion}

Clinical manifestations of this dog were atypical. Lameness severity has been reported to be related to OCD fragment size and to the severity of synovitis caused by joint instability. ${ }^{13,19}$ However, the patient described here had relatively modest lameness considering the notable size of the osteochondral fragment. The Golden Retriever is one of the breeds 
most frequently affected by OCD of the lateral trochlear ridge. ${ }^{18}$ The patient was female, in accordance with the gender predisposition reported in the literature. ${ }^{6}$

Radiography is reported being the first diagnostic investigation to be performed in case of OCD suspicion in the dog. ${ }^{20}$ Radiographic survey should include the following projections: mediolateral extended, mediolateral flexed, axial flexed craniocaudal, plantar-dorsal, plantar medialdorsal-lateral and plantar lateral-dorsal-medial oblique. $^{20,21}$ The dorsal-plantar projection provides the best diagnostic information for lesions of the dorsal aspect of the medial talar ridge, while the plantarolateral-dorsomedial $45^{\circ}$ oblique projection provides the best diagnostic information of the lateral ridge. ${ }^{20}$ The X-rays did not enable to diagnose OCD with certainty in the case here described, so CT was performed to better quantify the size and locate the fragment more accurately. Despite a detailed description of the canine tarsal radiographic anatomy, ${ }^{22}$ because of the superimposition tibia, fibula and calcaneus, radiographic images can be challenging to be interpreted. Computed tomography has been found to be superior to radiography for OCD diagnosis ${ }^{21}$. Computed tomography images showed an image suggestive of OCD on the lateroproximal aspect of the lateral ridge of the talar trochlea. The measured fragment was $10 \times 6 \times 5 \mathrm{~mm}$. A slight joint space narrowing and a substantial decrease in bone density were observed (-Fig. 3). These findings are compatible with moderate osteopenia, likely secondary to disuse. Fragment borders were partially rounded, suggesting the chronic nature of the condition. In addition, moderate periarticular soft-tissue swelling and mild talar-calcaneal arthritis were detected probably because of the joint instability.

Ultrasonography was useful for detecting joint effusion and for showing the subchondral bone fracture on the lateral side of the talar trochlea. Ultrasonography has been found to be a valid diagnostic imaging technique for the evaluation of bone surfaces. ${ }^{23}$ About $75 \%$ of the talar trochlear margin can be visualized by ultrasonography and the areas that can be seen are those most commonly affected by OCD. ${ }^{23}$ Ultrasonography, in addition to radiographs and CT images, could increase the accuracy of tarsal OCD lesions evaluations.

According to canine tarsal OCD literature, important prognostic information are provided by the fragment size. ${ }^{20}$ Removal of large osteochondral fragments usually results in severe incongruency, joint instability osteoarthritis and lameness. ${ }^{3,12}$ In this patient, given the size of the OCD fragment, fragment removal was not considered as an option. The pantarsal arthrodesis was then performed. The reported requisites for a successful tibiotarsal pan-arthrodesis include a rigid fixation system, as well as a complete approach to the joint surfaces and meticulous removal of the cartilage. ${ }^{18}$ Angular fixation systems provide greater and longer-lasting stability than conventional fixation systems. ${ }^{2}$ This system was also chosen based on its feature of being already bent at an angle of $135^{\circ}{ }^{24}$ This shortened the surgery time.

Conventional screws were used to achieve compression between the surfaces of the plate and the bone, thereby reducing implant-bone offset and enabling better soft tissues apposition. Bone healing was assessed on radiographic findings, which did not show any radiolucency of the juxtaposed bone surfaces and on the patient's clinical findings as no pain upon palpation. Implants were removed as at 7-month X-ray follow-up osteolysis around the screws and areas of bone radiolucency were detected. These findings were interpreted as stress-protection phenomena (-Fig. 4). ${ }^{25}$ The dog had developed a florid, large bone callous at the tibial extremity of the plate, incorporating it and greatly increasing distal tibia thickness (-Fig. 3). This exuberant callous could have been the result of excessive flexibility of the plate, or a consequence of the age (young) of the dog or both.

The expected healing time for an arthrodesis of the tarsus is 2 to 3 months. ${ }^{26}$ The rapid healing in the case reported here could have been facilitated by the patient's young age, the stability afforded by a locked system and/or the presence of abundant heterologous transplant material. ${ }^{27}$ In humans, healing time can be shortened by using biomaterials in cases of trauma, tumours or corrective osteotomies. ${ }^{27}$

Fragment histological examination determined that the joint fragment was a piece of cartilage with no signs of calcification or vascularization, leading to the histological diagnosis of hock OCD (-Fig. 5).

The tibiotarsal pan-arthrodesis clearly improved the patient's quality of life, despite the animal having a persistent lameness, which could be defined as a mechanical lameness with no pain, caused by joint immobility.

Numerous complications of tibiotarsal pan-arthrodesis have been reported in the literature, including calcaneal fractures, distal limb swelling, implant failure, infection, angular deformities, persistent lameness, wound dehiscence and plantar necrosis. ${ }^{18}$ No complications were noted in this case. On the basis of this experience and with all the caveats related to the evaluation of a single case, the authors consider that pan-arthrodesis is a surgical therapeutic possibility in cases of hock full-thickness OCD lesions which could cause severe joint instability and, osteoarthritis, thereby negatively affecting patient's long-term life quality.

\section{Author Contribution}

Petazzoni Massimo and Buiatti Michela contributed to study design, acquisition of data and data analysis and interpretation. Petazzoni Massimo also contributed to conception of study. Both authors drafted, revised and approved the submitted manuscript.

\section{Conflict of Interest}

None declared.

\section{Acknowledgments}

We thank Dr. Davide Zani, Dr. Mauro Gincamillo and Dr. Maurizio Longo for the computed tomography examination and Dr. Daniela Olivero for the histological studies.

\section{References}

1 Olson SE. Pathophysiology, morphology, and clinical signs of osteochondrosis in the dog. In: Bojarab MJ, ed. Disease Mechanisms in Small Animal Surgery. Philadelphia: Lea \& Febiger; 1993:777-796 
2 Demko J, McLaughlin R. Developmental orthopedic disease. Vet Clin North Am Small Anim Pract 2005;35(05):1111-1135, v

3 Martinez SA. Congenital conditions that lead to osteoarthritis in the dog. Vet Clin North Am Small Anim Pract 1997;27(04):735-758

4 Montgomery RD, Hathcock JT, Milton JL, Fitch RB. Osteochondritis dissecans of the canine tarsal joint. Compend Contin Educ Pract Vet 1994;16:835-845

5 Mortellaro CM, Petazzoni M, Vezzoni A. Atlante BOA. Milano: Innovet; 2008:63-64

6 Breur GJ, Spaulding KA, Braden TD. Osteochondritis dissecans of the medial trochlear ridge of the talus in the dog. Vet Comp Orthop Traumatol 1989;4:168-176

7 La Fond E, Breur GJ, Austin CC. Breed susceptibility for development orthopedic diseases in dogs. J Am Anim Hosp Assoc 2002;38 (05):467-477

8 Brinker WO, Piermattei DL, Flo GL. Diagnosis and treatment of orthopedic conditions of the forelimb. In: Brinker WO, Piermattei DL, Flo GL, eds. Brinker, Piermattei, and Flo's Handbook of Small Animal Orthopedics and Fracture Treatment. 2nd ed. Philadelphia: WB Saunders; 1990:486-490

9 Probst CW, Johnston SA. Osteochondrosis. In: Slatter DH, ed. Textbook of Small Animal Surgery. Philadelphia: WB Saunders; 1993:1944-1966

10 Olson NC, Mostosky UV, Flo GL, Tvedten HW. Osteochondritis dissecans of the tarsocrural joint in three canine siblings. J Am Vet Med Assoc 1980;176(07):635-637

11 Miller J, Beale B. Tibiotarsal arthroscopy. Applications and long-term outcome in dogs. Vet Comp Orthop Traumatol 2008;21(02):159-165

12 van der Peijl GJ, Schaeffer IG, Theyse LF, Dijkshoorn NA, Schwencke M, Hazewinkel HA. Osteochondrosis dissecans of the tarsus in Labrador Retrievers: clinical signs, radiological data and force plate gait evaluation after surgical treatment. Vet Comp Orthop Traumatol 2012;25(02):126-134

13 Egan P, Murphy S, Jovanovik J, Tucker R, Fitzpatrick N. Treatment of Osteochondrosis Dissecans of the Canine Stifle Using Synthetic Osteochondral Resurfacing. Vet Comp Orthop Traumatol 2018;31 (02):144-152

14 Welch JA. The tarsus and metatarsus. In: Slatter DH, ed. Textbook of Small Animal Surgery. 4th ed. Philadelphia, PA: WB Saunders; 1993:2158-2167
15 Maley JR, Dvorak LD, Bahr A. Diagnosis and management of a fracture of the lateral trochlear ridge of the talus in a dog. Vet Comp Orthop Traumatol 2010;23(04):284-288

16 Marshall GW, Dee JF. Repair of fractures of the tarsus. In: Bojrab MJ, ed. Current techniques in small animal surgery. 5th ed. Baltimore, MD: Williams and Wilkins; 2014:1105-1112

17 Lesser AS. Arthrodesis. In: Slatter D, ed. Textbook of Small Animal Surgery. 2nd ed. Philadelphia: WB Saunders; 1993:1888-1901

18 Roch SP, Clements DN, Mitchell RA, et al. Complications following tarsal arthrodesis using bone plate fixation in dogs. J Small Anim Pract 2008;49(03):117-126

19 Gielen I, Van Ryssen B, Coopman F, van Bree H. Comparison of subchondral lesion size between clinical and non-clinical medial trochlear ridge talar osteochondritis dissecans in dogs. Vet Comp Orthop Traumatol 2007;20(01):8-11

20 Kippenes H, Johnston G. Diagnostic imaging of osteochondrosis. Vet Clin North Am Small Anim Pract 1998;28(01):137-160

21 Gielen I, van Ryssen B, van Bree H. Computerized tomography compared with radiography in the diagnosis of lateral trochlear ridge talar osteochondritis dissecans in dogs. Vet Comp Orthop Traumatol 2005;18(02):77-82

22 Carlisle CH, Reynolds KM. Radiographic anatomy of the tarsocrural joint of the dog. J Small Anim Pract 1990;31:273-279

23 Liuti T, Saunders JH, Gielen I, De Rycke L, Coopman F, van Bree $\mathrm{H}$. Ultrasound approach to the canine distal tibia and trochlear ridges of the talus. Vet Radiol Ultrasound 2007;48(04): 361-367

24 Petazzoni M, Urizzi A, Verdonck B, Jaeger G. Fixin internal fixator: concept and technique. Vet Comp Orthop Traumatol 2010;23(04): 250-253

25 Petazzoni M, Nicetto T, Urizzi A. Bone resorption secondary to suspected stress protection using a locking plate. Veterinaria. 2012;26(02):31-36

26 Lesser AS. Tibiotarsal arthrodesis and other tarsal arthrodesis procedures. In: Bojrab MJ, ed. Current Techniques in Small Animal Surgery. 5th ed. Baltimore, MD: Williams and Wilkins; 2014:1114-1118

27 Wee J, Thevendran G. The role of orthobiologics in foot and ankle surgery: allogenic bone grafts and bone graft substitutes. EFORT Open Rev 2017;2(06):272-280 